\title{
Les variations individuelles de la lipolyse spontanée du lait de vache : effet du numéro de lactation et répétabilité au cours de deux lactations successives
}

\author{
Marie-Paule CHAZAL ${ }^{(1)}$, Y. CHILLIARD ${ }^{(2)}$ \\ INRA, Laboratoire de la Lactation, Theix, 63122 Ceyrat, France
}

Résumé

L'effet du numéro de lactation sur les teneurs en acides gras libres du lait de vache après la traite (AGL initiaux) et après $22 \mathrm{~h}$ de stockage à $4^{\circ} \mathrm{C}$ (AGL22) a été étudié en hiver et au printemps, durant 2 années successives, sur 162 à 267 vaches de 3 ou 4 troupeaux expérimentaux. Les teneurs en AGL22 des laits individuels tendent à diminuer avec l'âge des animaux, mais seulement au printemps lorsque les animaux sont à des stades de lactation et de gestation plus avancés qu'en hiver. Toutefois, le suivi des mêmes animaux d'une lactation à la suivante n'a pas confirmé cette tendance.

La répétabilité entre deux lactations successives de la teneur en AGL22 du lait a été étudiée sur 111 vaches en hiver, et 131 vaches au printemps, toutes en milieu de lactation et en début de gestation. Elle explique alors 30 à $40 \%$ de la variance totale des données. Chez 35 vaches d'un troupeau suivi régulièrement au cours de 2 lactations successives, l'augmentation des teneurs en AGL22 en fin de gestation, lorsqu'elle a lieu, semble être à la fois sous la dépendance d'un facteur intrinsèque répétable d'une lactation à la suivante, et d'un facteur extrinsèque, non répétable et lié probablement aux conditions alimentaires.

Mots clés : Lait de vache - Lipolyse spontanée - Acidité de la matière grasse - Numéro de lactation - Variations individuelles - Répétabilité entre lactations.

\section{Summary}

Individual variations in spontaneous lipolysis in bovine milk : effect of lactation number, and repeatability during two consecutive lactations

The effect of lactation number on milk free fatty acid contents (FFA) after milking (initial FFA), and after $22 \mathrm{~h}$ storage at $4^{\circ} \mathrm{C}$ (FFA-22) has been studied during two successive years $(1984,1985)$ on 162 to 267 cows, in winter, when all cows were kept indoors, and in spring at pasture.

(1) Institut Technique de l'Elevage Bovin.

(2) Correspondance et demandes de tirés-à-part. 
In winter, milk initial FFA content tended to increase $(\mathrm{P}<0.05)$ with lactation number. In spring, its variation was lower than that in winter and was not related to lactation number. Initial FFA were lower in 1985 than in $1984(\mathrm{P}<0.05)$. Milk initial FFA contents of the two successive years at the same season were not well correlated ( $\mathrm{r}=0.27, \mathrm{n}=111$, in winter, and $\mathrm{r}=0.11, \mathrm{n}=131$, in spring).

Milk FFA-22 content varied non significantly with lactation number in winter during both years. But in spring (or during late lactation) it decreased when number of lactation increased $(\mathrm{P}<0.05)$. However, when the same cows were studied during two successive spring, no decrease was found from one lactation to the next. The " lactation number » effect could have been concealed by a « year » effect.

Repeatability of milk FFA-22 contents between two consecutive lactations was studied on 111 cows in winter and 131 cows in spring, all the cows being in midlactation and in early pregnancy or non-pregnant. Milk FFA-22 contents during two successive years were highly correlated $(\mathrm{r}=0.55, \mathrm{n}=111$, in winter, and $\mathrm{r}=0.64$, $\mathrm{n}=131$, in spring). This correlation was not due to differences in milk somatic cell counts between cows.

Twenty-seven cows were studied regularly during two successive lactations, up to at least 28 weeks of pregnancy. Among 16 cows whose milk FFA-22 content increased during late prenancy in 1984, 10 cows had the same lipolytic pattern in 1985 . The 6 other cows produced milk the FFA-22 content of which did not increase during late pregnancy in 1985. In the eleven other cows, milk FFA-22 remained low during both years. Thus, the increase of milk FFA-22 content during late pregnancy, if it occurs, seems to be dependent on both an intrinsic factor repeatable from one lactation to another, and an extrinsic factor probably linked to feeding conditions.

Key words: Bovine milk - Spontaneous lipolysis - Fat acidity - Number of lactation Individual variations - Repeatability between consecutive lactations.

\section{Introduction}

La lipolyse spontanée du lait refroidi est l'hydrolyse enzymatique de sa matière grasse, en absence de toute activation mécanique ou thermique. Elle dépend d'un certain nombre de facteurs tels que les stades de lactation et de gestation, et le niveau de production (HunTER et al., 1968; OrTiz et al., 1970 ; Jellema, 1975 ; Ahrné et Buörck, 1985 ; Chazal et Chilliard, 1986, 1987a). Toutefois, à même stade physiologique et à même niveau de production, la lipolyse spontanée présente une grande variabilité entre individus. Cet effet individuel pourrait être répétable d'une lactation à l'autre chez certains animaux (KuzDzal-Savoie et Mocquot, 1960). Il n'est pas expliqué par la race (Herrington et Krukovsky, 1939; Roahen et Sommer, 1940 ; Chazal et Chilliard, 1987b). Cependant un effet génétique intrarace a été suggéré (Deeth et Fitz-Gerald, 1976), bien qu'il n'ait pas été mis en évidence par WINTER et al. (1981). L'effet du numéro de lactation n'a été que peu étudié : la lipolyse spontanée semble plus élevée chez les primipares, d'après ConNOLLY (1978), ou chez les multipares à partir de la $7^{\mathrm{e}}$ lactation, d'après KielszNiA et KrolikowSKa (1982).

Compte tenu de la part importante des variations individuelles dans les variations physiologiques de la lipolyse, et du faible nombre d'études recherchant leurs causes, l'objet de ce travail a été de mesurer l'effet du numéro de lactation sur la lipolyse spontanée et la répétabilité de celle-ci d'une lactation à la suivante. 


\section{Matériel et méthodes}

\section{A. Animaux}

862 prélèvements de lait ont été réalisés, au cours d'une ou deux années, sur les animaux de 4 troupeaux expérimentaux : 2 troupeaux INRA (Marcenat, Cantal ; Theix, Puy-de-Dôme) et 2 troupeaux en convention avec I'ITEB (Lycée Agricole de Bourg-en-Bresse, Ain ; ferme expérimentale de La CôteSaint-André, EDE de l'Isère). Un prélèvement de lait a été effectué sur chaque vache en fin de période hivernale, les animaux étant à l'étable et recevant de l'ensilage de maïs seul (Theix, La Côte-Saint-André) ou avec de l'ensilage de luzerne (La Côte-Saint-André), de l'ensilage de maïs et de l'ensilage d'herbe (Bourg-en-Bresse) ou de l'ensilage d'herbe et du foin (Marcenat). Un $2^{\mathrm{e}}$ prélèvement a été effectué sur chaque vache, au printemps, au pâturage. En période hivernale, les animaux recevaient du tourteau de soja ou de colza en complément de l'ensilage de maïs (Theix, La Côte-Saint-André). Une partie des animaux de Bourg-en-Bresse recevait du lactosérum et l'ensemble des drêches de brasserie. Les animaux des 4 troupeaux recevaient aussi de l'aliment concentré en complément de la ration de base.

Le nombre de vaches, leur répartition par troupeau et par numéro de lactation ainsi que leurs caractéristiques physiologiques (stades de lactation et de gestation, niveau de production à la traite du soir) sont présentés dans le tableau 1. Des animaux de trois troupeaux ont été suivis durant 2 années successives (Marcenat, Theix, La Côte-Saint-André), soient 111 vaches en fin de période hivernale à l'étable, et 131 vaches au printemps, au pâturage, à des stades de lactation comparables d'une année à l'autre (la différence entre les stades de lactation d'une année à l'autre est de $1 \pm 5$ semaines, à la fois en hiver et au printemps).

\section{B. Prélèvements de lait et analyse des échantillons}

Le lait était prélevé dans le pot trayeur (troupeau de Theix en période hivernale et de Marcenat) ou dans un compteur à lait de marque Vaïkato placé juste après la griffe (troupeau de Theix en période de pâturage, de Bourg-en-Bresse et de La Côte-Saint-André). Les échantillons ont été traités selon les méthodes décrites par Chazal et Chilliard $(1986,1987 a)$ en vue du dosage des acides gras libres (AGL) après la traite (AGL initiaux) et après un stockage de $22 \mathrm{~h}$ à $4{ }^{\circ} \mathrm{C}$ (AGL22), par une variante miniaturisée de la méthode BDI (Bureau of Dairy Industry) (ChazAl et al., 1984)

\section{Analyse des résultats}

Deux aspects ont été étudiés :

- l'effet du numéro de lactation sur les teneurs en $A G L$ du lait : l'analyse a été réalisée en comparant les laits individuels à chacune des 4 périodes de prélèvement. Une analyse de variance et de covariance (Seebeck, 1973) a été effectuée après transformation logarithmique des teneurs en AGL, afin d'obtenir une distribution normale des données. L'analyse a pris en compte les 2 
TABleau 1

Répartition des animaux selon le troupeau et le numéro de lactation et caractéristiques des prélèvements de lait

(Moyenne et écart-type entre parenthèses)

Distribution of the animals according to herd and lactation number and characteristics of the milk samples (mean and s.d. in brackets)

\begin{tabular}{|c|c|c|c|c|}
\hline $\begin{array}{l}\text { Année } \\
\text { Year }\end{array}$ & \multicolumn{2}{|c|}{1984} & \multicolumn{2}{|c|}{1985} \\
\hline $\begin{array}{l}\text { Période } \\
\text { Period }\end{array}$ & $\begin{array}{l}\text { Hiver } \\
\text { Winter }\end{array}$ & $\begin{array}{l}\text { Printemps } \\
\text { Spring }\end{array}$ & $\begin{array}{l}\text { Hiver } \\
\text { Winter }\end{array}$ & $\begin{array}{l}\text { Printemps } \\
\text { Spring }\end{array}$ \\
\hline $\begin{array}{l}\text { Nombre de vaches } \\
\text { Cow number }\end{array}$ & 236 & 267 & 162 & 197 \\
\hline $\begin{array}{l}\text { Répartition par troupeaux } \\
\text { Herd distribution } \\
\text { Bourg-en-Bresse } \\
\text { Marcenat } \\
\text { La Côte Saint-André } \\
\text { Theix }\end{array}$ & $\begin{array}{l}43 \\
90 \\
56 \\
47\end{array}$ & $\begin{array}{l}44 \\
99 \\
55 \\
69\end{array}$ & $\begin{array}{l}- \\
89 \\
55 \\
18\end{array}$ & $\begin{array}{l}- \\
96 \\
42 \\
59\end{array}$ \\
\hline $\begin{array}{l}\text { Répartition par numéro } \\
\text { de lactation } \\
\text { Lactation number distribution } \\
1 \\
2 \\
3 \\
\geqslant 4\end{array}$ & $\begin{array}{l}67 \\
75 \\
19 \\
75\end{array}$ & $\begin{array}{l}90 \\
79 \\
19 \\
79\end{array}$ & $\begin{array}{l}51 \\
35 \\
36 \\
40\end{array}$ & $\begin{array}{l}65 \\
48 \\
36 \\
48\end{array}$ \\
\hline $\begin{array}{l}\text { Stade de lactaction (semaines) } \\
\text { Stage of lactation (weeks) }\end{array}$ & $16(6)$ & $28(8)$ & $19(6)$ & $30(7)$ \\
\hline $\begin{array}{l}\text { Stade de gestation (semaines) } \\
\text { Stage of pregnancy (weeks) }\end{array}$ & $7(4)$ & $13(9)$ & $7(6)$ & $16(7)$ \\
\hline $\begin{array}{l}\text { Production à la traite du soir } \\
(\mathrm{kg}) \\
\text { Evening milk yield }(\mathrm{kg})\end{array}$ & $8,8(2,6)$ & $7,6 \quad(2,5)$ & $7,8 \quad(2,3)$ & $6,8 \quad(1,9)$ \\
\hline $\begin{array}{l}\text { AGL initiaux (meq/100 g MG) } \\
\text { Initial FFA (meq/100 g fat) }\end{array}$ & $0,25(0,06)$ & $0,29(0,08)$ & $0,21(0,07)$ & $0,18(0,06)$ \\
\hline $\begin{array}{l}\text { AGL22 (meq/100 g MG) } \\
F F A-22 \text { (meq/100 g fat) }\end{array}$ & $0,62(0,30)$ & $0,70(0,35)$ & $0,65(0,35)$ & $0,74(0,49)$ \\
\hline
\end{tabular}


facteurs «numéro de lactation» et «troupeau». Les covariables étaient le stade de lactation, le stade de gestation et le niveau de production, compte tenu de leurs effets sur la lipolyse spontanée (Chazal et Chilliard, 1986, 1987a). Les données du tableau 2 sont les moyennes ajustées. L'effet de l'âge des animaux a également été étudié en comparant, pour un même animal et à stades physiologiques comparables (cf. tabl. 3), l'évolution des teneurs en AGL du lait de 92 vaches au cours de 2 lactations successives (analyse de variance par couples) ;

- la répétabilité des teneurs en AGL du lait entre lactations successives a été évaluée sur 3 troupeaux, en période hivernale et en période de pâturage, en établissant les corrélations entre les teneurs en AGL du lait de 2 lactations successives (données non transformées), les animaux étant en moyenne en milieu de lactation et en début de gestation. Elle a également été étudiée en fin de gestation, chez 35 vaches gravides du troupeau de La Côte-Saint-André.

\section{Résultats}

\section{A. Teneurs en AGL initiaux du lait}

En fin de période hivernale les teneurs en AGL initiaux du lait tendent à augmenter avec le numéro de lactation des animaux $(P<0,05)$, ceci de façon plus nette en 1985 qu'en 1984. Au pâturage, les variations de ces teneurs sont plus faibles qu'en période hivernale et ne sont pas ordonnées selon le numéro de lactation (tabl. 2). Les teneurs en AGL initiaux sont plus faibles en 1985 qu'en 1984 (tabl. 2 et 3). Mesurées au cours de 2 lactations successives, elles sont faiblement corrélées, que ce soit en hiver à l'étable $(r=0,27, \mathrm{n}=111)$ ou au printemps au pâturage $(\mathrm{r}=0,11, \mathrm{n}=131)$.

\section{B. Teneurs en AGL22 du lait}

\section{Effet du numéro de lactation}

Les teneurs en AGL22 du lait varient peu avec le numéro de lactation durant les hivers 1984 et 1985. Par contre, aux printemps 1984 et 1985, on observe une diminution de ces teneurs avec l'âge des animaux, qui devient significative à partir de la $3^{\mathrm{c}}$ lactation $(\mathrm{P}<0,05)($ tabl. 2$)$. Il n'existe pas d'interaction significative entre les facteurs «numéro de lactation» et « troupeau ». Toutefois, même au printemps, cette diminution des teneurs en AGL22 est faible d'une lactation à la suivante pour un même animal. Elle n'est significative que dans les laits des animaux passant de la lactation 2 à la lactation $3(\mathrm{P}<0,05)($ tabl. 3), et résulte de celle des teneurs en AGL initiaux dans le lait des mêmes animaux.

\section{Répétabilité entre lactations successives}

Les teneurs en AGL22 du lait des vaches de 3 troupeaux en milieu de lactation mesurées respectivement au cours de 2 lactations successives sont assez bien corrélées $(\mathrm{r}=0,55, \mathrm{n}=111$ en hiver, et $\mathrm{r}=0,64, \mathrm{n}=131$ au 


\section{Tableau 2}

Effet du numéro de lactation sur les teneurs en acides gras libres du lait avant (AGL initiaux) et après $22 \mathrm{~h}$ de stockage à $4{ }^{\circ} \mathrm{C}$ (AGL22) ${ }^{(1)}$

Effect of lactation number on the free fatty acid contents in milk before (Initial FFA) and after $22 \mathrm{~h}$ storage at $4^{\circ} \mathrm{C}(F F A-22)^{(1)}$

\begin{tabular}{|c|c|c|c|c|c|}
\hline \multicolumn{2}{|c|}{$\begin{array}{l}\text { Numéro de lactation } \\
\text { Number of lactation }\end{array}$} & 1 & 2 & 3 & $\geqslant 4$ \\
\hline \multirow{4}{*}{$\begin{array}{l}\text { AGL initiaux } \\
\text { Initial } F F A^{(2)}\end{array}$} & $\begin{array}{l}\text { Hiver } 1984 \\
\text { Winter } 1984\end{array}$ & $0,21^{\mathrm{a}}$ & $0,26^{\mathrm{b}}$ & $0,24^{b}$ & $0,26^{\mathrm{b}}$ \\
\hline & $\begin{array}{l}\text { Hiver } 1985 \\
\text { Winter } 1985\end{array}$ & $0,17^{\mathrm{a}}$ & $0,18^{\mathrm{a}}$ & $0,21^{\mathrm{b}}$ & $0,23^{\mathrm{b}}$ \\
\hline & $\begin{array}{l}\text { Printemps } 1984 \\
\text { Spring } 1984\end{array}$ & $0,26^{\mathrm{a}}$ & $0,29^{\mathrm{b}}$ & $0,26^{\mathrm{a}}$ & $0,29^{\mathrm{b}}$ \\
\hline & $\begin{array}{l}\text { Printemps } 1985 \\
\text { Spring } 1985\end{array}$ & 0,18 & 0,20 & 0,18 & 0,19 \\
\hline \multirow{4}{*}{$\begin{array}{l}\text { AGL22 (2) } \\
F F A-22^{(2)}\end{array}$} & $\begin{array}{l}\text { Hiver } 1984 \\
\text { Winter } 1984\end{array}$ & $0,53^{\mathrm{ab}}$ & $0,61^{\mathrm{a}}$ & $0,47^{b}$ & $0,56^{\mathrm{ab}}$ \\
\hline & $\begin{array}{l}\text { Hiver } 1985 \\
\text { Winter } 1985\end{array}$ & 0,56 & 0,60 & 0,65 & 0,61 \\
\hline & $\begin{array}{l}\text { Printemps } 1984 \\
\text { Spring } 1984\end{array}$ & $0,72^{\mathrm{a}}$ & $0,67^{\mathrm{a}}$ & $0,51^{b}$ & $0,57^{\mathrm{b}}$ \\
\hline & $\begin{array}{l}\text { Printemps } 1985 \\
\text { Spring } 1985\end{array}$ & $0,79^{\mathrm{a}}$ & $0,69^{\mathrm{a}}$ & $0,51^{\mathrm{b}}$ & $0,58^{\mathrm{b}}$ \\
\hline \multicolumn{6}{|c|}{$\begin{array}{l}\text { (1) Voir tableau } 1 \text { pour les effectifs et les caractéristiques des vaches. } \\
\text { (1) See table } 1 \text { for number and characteristics of the cows. } \\
\text { (2) En meq/ } 100 \mathrm{~g} \text { MG. Moyennes ajustées après élimination de l'effet troupeau et en fonction des covariables : stade } \\
\text { de lactation, stade de gestation et niveau de production à la traite du soir. } \\
\text { (2) In meq I00 g fat. Mean values adjusted after removing of herd effect and according to covariates : stage of lactation, } \\
\text { stage of pregnancy and evening milk yield. } \\
\text { a, b : des résultats indicés par des lettres différentes sur une même ligne sont significativement différents }(\mathrm{P}<0,05) \text {. } \\
a, b \text { : values in the same horizontal row with different superscritps differ significantly }(P<0.05) \text {. }\end{array}$} \\
\hline
\end{tabular}

printemps, $\mathrm{P}<0,001$ ) (fig. 1). La variation résiduelle reste toutefois importante (de 60 à $70 \%$ de la variance totale) mais n'est que peu liée au stade de lactation ou au niveau de production, compte tenu des faibles corrélations entre les teneurs en AGL22 et ces deux paramètres (coefficients de corrélation compris entre 0,14 et 0,32 pour 111 à 131 données durant les 2 hivers et les 2 printemps 1984 et 1985). 
TABLEAU 3

Teneurs en acides gras libres du lait de vache avant ( $A G L$ initiaux) et après $22 \mathrm{~h}$ de stockage à $4{ }^{\circ} \mathrm{C}$ (AGL22) au cours de deux lactations consécutives (prélèvements de printemps)

Free fatty acid contents in bovine milk before (Initial FFA) and after $22 \mathrm{~h}$ storage at $4{ }^{\circ} \mathrm{C}$ (FFA-22) during two consecutive years (spring samples)

\begin{tabular}{|c|c|c|c|c|c|c|}
\hline \multirow{2}{*}{$\begin{array}{c}\text { Nombre de vaches } \\
\text { Cow number } \\
\text { Année } \\
\text { Year }\end{array}$} & \multicolumn{2}{|c|}{47} & \multicolumn{2}{|c|}{35} & \multicolumn{2}{|c|}{10} \\
\hline & 1984 & 1985 & 1984 & 1985 & 1984 & 1985 \\
\hline $\begin{array}{l}\text { Numéro de lactation } \\
\text { Number of lactation }\end{array}$ & 1 & 2 & 2 & 3 & 3 & 4 \\
\hline $\begin{array}{l}\text { AGL initiaux (meq/100 g MG) } \\
\text { Initial FFA (meq/100 } \mathrm{g} \text { fat })\end{array}$ & $\begin{array}{l}0,27^{\mathrm{a}} \\
(0,08)\end{array}$ & $\begin{array}{l}0,19^{b} \\
(0,07)\end{array}$ & $\begin{array}{l}0,27^{\mathrm{a}} \\
(0,06)\end{array}$ & $\begin{array}{l}0,21^{\mathrm{b}} \\
(0,09)\end{array}$ & $\begin{array}{l}0,26^{\mathrm{a}} \\
(0,04)\end{array}$ & $\begin{array}{l}0,15^{\mathrm{b}} \\
(0,04)\end{array}$ \\
\hline $\begin{array}{l}\text { AGL22 (meq/100 g MG) } \\
\text { FFA-22 (meq/100 g fat) }\end{array}$ & $\begin{array}{c}0,80 \\
(0,34)\end{array}$ & $\begin{array}{c}0,75 \\
(0,48)\end{array}$ & $\begin{array}{l}0,57^{\mathrm{a}} \\
(0,19)\end{array}$ & $\begin{array}{l}0,50^{\mathrm{b}} \\
(0,21)\end{array}$ & $\begin{array}{c}0,47 \\
(0,10)\end{array}$ & $\begin{array}{c}0,43 \\
(0,19)\end{array}$ \\
\hline $\begin{array}{l}\text { Stade de gestation (semaines) } \\
\text { Stage of pregnancy (weeks) }\end{array}$ & $\begin{array}{l}16 \\
(5)\end{array}$ & $\begin{array}{l}15 \\
(7)\end{array}$ & $\begin{array}{l}12 \\
(7)\end{array}$ & $\begin{array}{l}11 \\
(7)\end{array}$ & $\begin{array}{c}9 \\
(7)\end{array}$ & $\begin{array}{l}10 \\
(9)\end{array}$ \\
\hline $\begin{array}{l}\text { Production à la traite du soir }(\mathrm{kg}) \\
\text { Evening milk yield }(\mathrm{kg})\end{array}$ & $\begin{array}{c}6,5 \\
(1,3)\end{array}$ & $\begin{array}{c}6,7 \\
(1,8)\end{array}$ & $\begin{array}{c}8,3 \\
(2,6)\end{array}$ & $\begin{array}{c}8,5 \\
(1,8)\end{array}$ & $\begin{array}{l}9,4^{\mathrm{a}} \\
(2,5)\end{array}$ & $\begin{array}{l}7,3^{\mathrm{b}} \\
(1,8)\end{array}$ \\
\hline
\end{tabular}

(1) 47 vaches dont 8 de La Côte Saint-André, 23 de Marcenat et 16 de Theix ; 35 vaches dont 18 de La Côte SaintAndré, 14 de Marcenat et 3 de Theix : 10 vaches de Marcenat.

(I) 47 cows : 8 from La Côte Saint-André, 23 from Marcenat, 16 from Theix ; 35 cows : 18 from La Côte Saint-André, 14 from Marcenat, 3 from Theix; 10 cows from Marcenat.

(2) Moyenne et écart-type entre parenthèses.

(2) Mean and s.d. in brackets.

$\mathrm{a}, \mathrm{b}$ : des résultats indicés par des lettres différentes sont significativement différents $(\mathrm{P}<0,05)$.

$a, b:$ values with different superscritps differ significantly $(P<0.05)$.

Sur le troupeau de La Côte-Saint-André, les teneurs en AGL22 du lait de 35 vaches gravides depuis au moins 20 semaines ont été comparées durant 2 années successives, à même stade de gestation (moyenne des écarts entre stades de $1 \pm 1$ semaine), et à niveau de production comparable (moyenne des écarts de production la traite du soir de $0,9 \pm 0,7 \mathrm{~kg}$ ) (fig. 2). Chez 29 vaches, les teneurs en AGL22 entre lactations successives sont bien corrélées $(\mathrm{r}=0,89)$. Chez 6 autres vaches, les teneurs en AGL22 du lait ont été élevées la $1^{\text {re }}$ année (de 2,0 à $7,8 \mathrm{meq} / 100 \mathrm{~g} \mathrm{MG}$ ), mais sont restées faibles la $2^{\mathrm{e}}$ année (de 0,4 à $1,1 \mathrm{meq} / 100 \mathrm{~g} \mathrm{MG}$ ). 


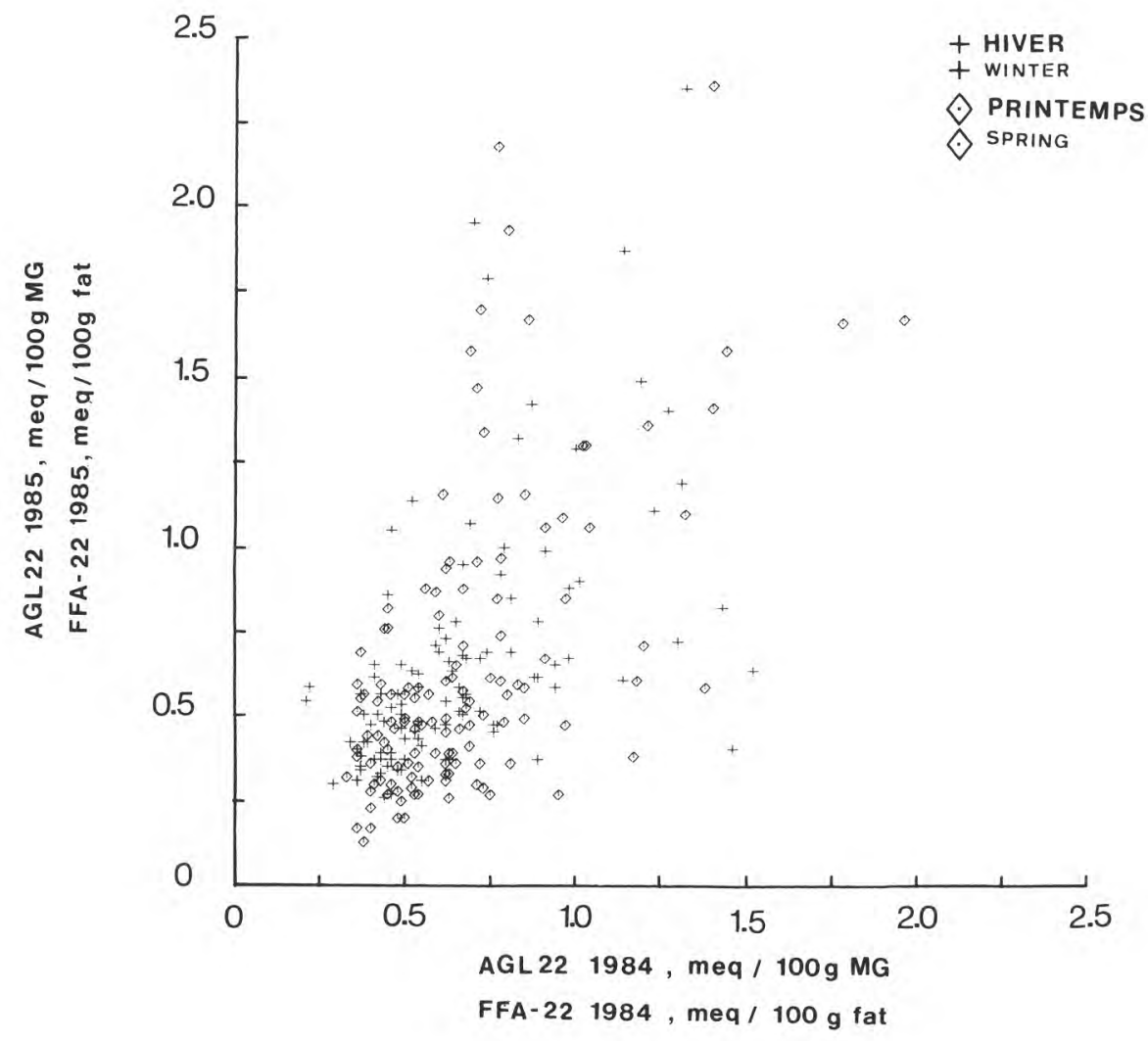

Fig. 1

Teneurs en acides gras libres du lait de vache après $22 \mathrm{~h}$ de stockage à $4{ }^{\circ} \mathrm{C}$ (AGL22), mesurées durant deux lactations consécutives.

Free fatty acid contents in bovine milk after $22 \mathrm{~h}$ storage at $4{ }^{\circ} \mathrm{C}$ (FFA-22) as measured during two consecutive lactations.

Nombre de vaches (La Côte Saint-André, Marcenat, Theix) : hiver, 111 (38-61-12) ; printemps, 131 (26-67-38).

Ecart entre les stades physiologiques des 2 années pour chaque animal:

- stade de lactation: hiver $1 \pm 5$ semaines; printemps $1 \pm 5$ semaines,

- stade de gestation: hiver $0 \pm 4$ semaines; printemps $1 \pm 7$ semaines.

Cow number (La Côte Saint-André, Marcenat, Theix) : winter, 111 (38-61-12) ; spring, 131 (26-67-38).

Difference between the physiological stages of the 2 years for each cow:

- stage of lactation: winter, $1 \pm 5$ weeks; spring, $1 \pm 5$ weeks,

- stage of pregnancy : winter, $0 \pm 4$ weeks; spring, $1 \pm 7$ weeks. 


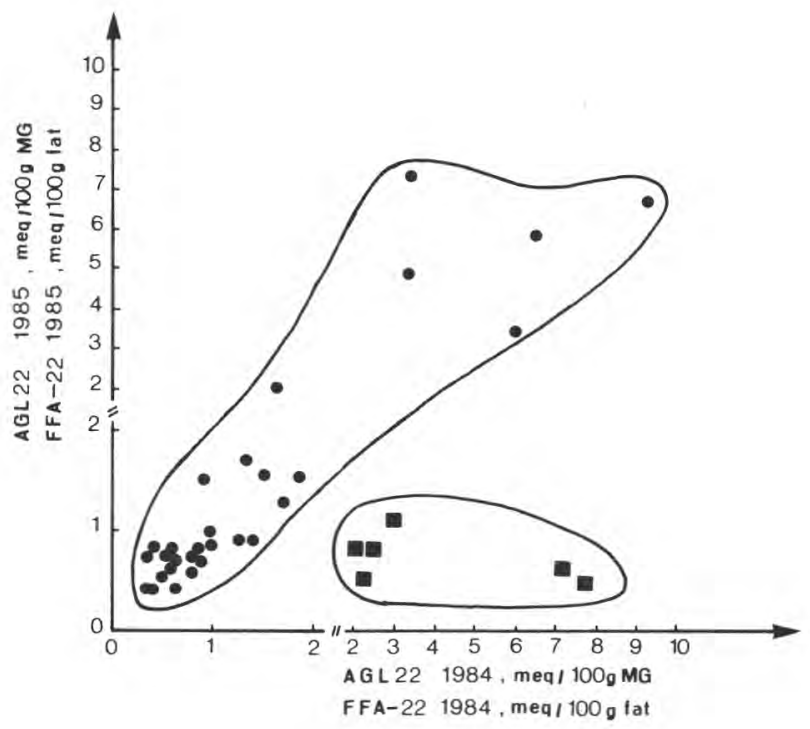

Fig. 2

Teneurs en acides gras libres après $22 \mathrm{~h}$ de stockage à $4{ }^{\circ} \mathrm{C}$ (AGL22) du lait de 35 vaches en fin de gestation ( $\geqslant 20$ semaines), au cours de 2 lactations consécutives.

Free fatty acid contents after 22 h storage at $4{ }^{\circ} \mathrm{C}$ (FFA-22) in milk of 35 cows after 20 weeks of pregnancy, during 2 consecutive lactations.

Les stades de gestation et les niveaux de production pour un même animal à la traite du soir sont comparables d'une année à l'autre : la différence entre les 2 stades de gestation est de $1 \pm 1$ semaine, et celle entre les deux niveaux de production est de $0,9 \pm 0,7 \mathrm{~kg}$.

- : vaches des groupes 1, 2, $3(n=29)$; : vaches du groupe $4(n=6)$.

Stages of pregnancy and evening milk yields from year to year were comparable for each cow: the difference between the 2 pregnancy stages was $1 \pm 1$ week, and that between the two milk yields was $0.9 \pm 0.7 \mathrm{~kg}$.

- : cows of groups 1, 2, $3(n=29)$; : cows of group $4(n=6)$.

Parmi ces 35 vaches, 27 ont été suivies jusqu'à 28 semaines de gestation au moins (fig. 3). Respectivement 6, 4 et 11 vaches ont produit du lait dont la teneur en AGL22 augmente fortement (groupe 1), modérément (groupe 2) ou n'a pas augmenté (groupe 3) en fin de gestation au cours des 2 lactations consécutives. Dans un $4^{\mathrm{e}}$ groupe de 6 vaches, la teneur en AGL22 du lait en fin de gestation a augmenté la $1^{\text {re }}$ année mais pas la seconde.

En d'autres termes, parmi les 16 vaches dont la teneur en AGL22 du lait a augmenté en fin de gestation en 1984 (groupes 1, 2 et 4), 10 ont eu un comportement identique en 1985, les 6 autres ayant produit du lait à faible teneur en AGL22 l'année suivante. En outre, le cas inverse (niveau d'AGL22 du lait en fin de gestation faible en 1984, et élevé en 1985) n'a pas été observé. 


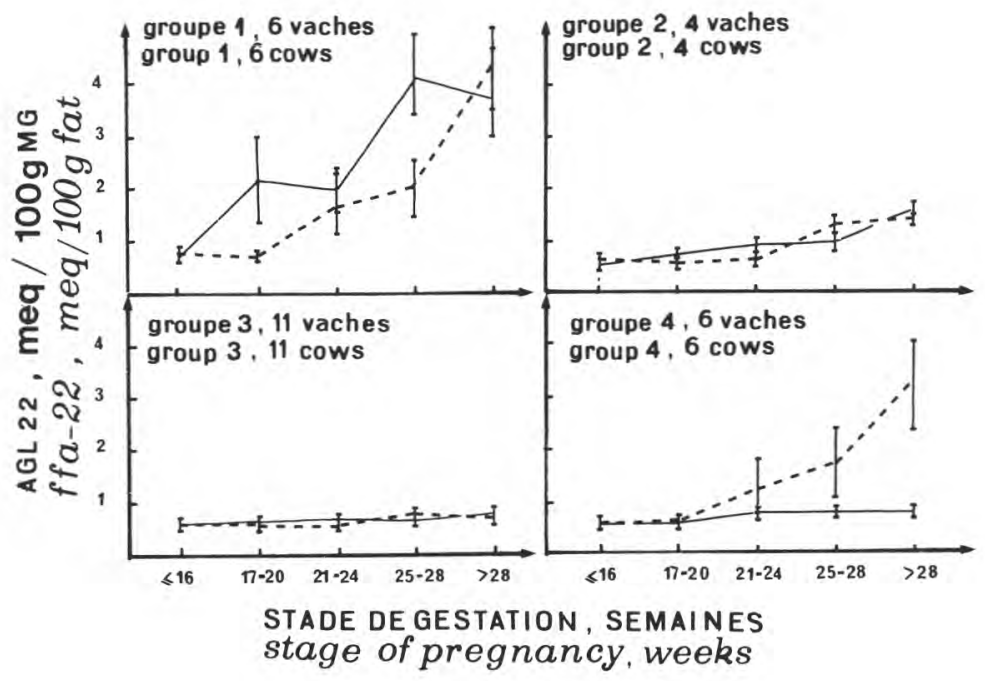

Fig. 3

Evolutions comparées au cours de 2 gestations consécutives des teneurs en acides gras libres du lait, après $22 \mathrm{~h}$ de stockage à $4{ }^{\circ} \mathrm{C}$ (AGL22).

Les valeurs moyennes sont représentées avec l'erreur-type

-. - année 1984 ; année 1985.

L'évolution des niveaux de production de lait à la traite du soir au cours de la gestation n'a pas été différente entre les 2 années et entre les 4 groupes de vaches.

Comparative evolutions of free fatty acid contents after $22 \mathrm{~h}$ storage at $4{ }^{\circ} \mathrm{C}$ (FFA-22) in bovine milk during two consecutive pregnancies.

Mean values are presented with standard-error - . -, year 1984 ; year 1985.

The evolution of evening milk yield during the pregnancy was not different between the 2 years and the 4 groups of cows.

\section{Discussion}

\section{A. Effet du numéro de lactation}

Bien que la teneur en AGL initiaux des laits soit liée positivement au taux de cellules (Tarassuk et Yaguchi, 1958 ; Tallamy et Randolph, 1969 ; Salih et Anderson, 1979 ; Chazal et Chilliard, 1987a), ce dernier ne peut pas être un facteur explicatif des teneurs en AGL initiaux plus faibles chez les primipares que chez les multipares. Par exemple, durant l'hiver 1984, le taux de cellules était identique pour les vaches jusqu'en troisième lactation (de 210 à $280 \times 10^{3}$ cellules $)$ et augmentait seulement à partir de la quatrième $\left(520 \times 10^{3}\right.$ cellules). 
Lorsque l'on compare les teneurs en AGL22 du lait des individus au cours d'une même année, celles-ci tendent à diminuer avec le numéro de lactation, en accord avec les résultats de Connolly (1978) qui observe des teneurs en AGL après stockage au froid plus élevées chez les primipares que chez les multipares, sans préciser les conditions expérimentales. Toutefois cette observation n'a été confirmée que sur les prélèvements de printemps. En hiver, les animaux sont en début de lactation et en début de gestation ou non gravides, et les teneurs en AGL22 sont plus faibles et moins variables qu'au printemps. Ainsi l'effet de l'âge pourrait être d'autant plus marqué que le stade de gestation est avancé. Ceci a été confirmé sur le troupeau de Theix, où le lait de 17 vaches (11 multipares et 6 primipares) a été prélevé non seulement au printemps 1984 mais aussi au cours de la dernière semaine avant la tarissement. Les teneurs en AGL22 des laits des primipares et des multipares ont été nettement plus différentes en fin de gestation $(2,81 \pm 2,01$ contre $0,97 \pm 0,47$ meq/100 g MG) qu'au printemps $(1,07 \pm 0,35$ contre $0,58 \pm 0,13 \mathrm{meq} / 100 \mathrm{~g}$ $\mathrm{MG})$.

Lorsque les teneurs en AGL22 du lait sont comparées pour une même vache au cours de 2 lactations successives sur 3 troupeaux (tabl. 3), l'effet de l'âge sur la lipolyse n'est par contre pas confirmé. Un effet « année » a peutêtre masqué l'effet "numéro de lactation». En effet, compte tenu de la diminution d'une année à l'autre des teneurs en AGL initiaux, la lipolyse (AGL22-AGL initiaux) a été plus élevée en 1985 qu'en 1984. Ces effets «année » pourraient être liés à d'éventuelles variations des conditions de prélèvement, de dosage, ou des conditions climatiques d'une année à l'autre.

Par ailleurs, on observe un niveau plus élevé en 1985 qu'en 1984 des teneurs en AGL22 des laits des vaches en seconde lactation (tabl. 3), qui dépend probablement de la répartition de ces vaches dans les différents troupeaux : en 1984, 17 vaches sur 35 (49\%) appartenaient aux troupeaux de Marcenat et de Theix, contre 39 sur 47 (83\%) en 1985. Or l'intervalle entre les traites du matin et du soir est plus court sur ces 2 exploitations (respectivement 9 h 30 et $8 \mathrm{~h} \mathrm{30)}$ qu'à La Côte-Saint-André $(10 \mathrm{~h})$, ce qui tend à augmenter les teneurs en AGL22 des laits des 2 premiers troupeaux à la traite du soir (Chazal et Chilliard, 1987a).

\section{B. Répétabilité au cours de 2 lactations successives}

Chez la chèvre l'activité lipasique et la lipolyse spontanée présentent une certaine répétabilité d'une lactation à la suivante chez un même animal (Chilliard et Morand-Fehr, 1978 ; Chilliard, 1982). Chez la vache, aucune étude comparant les teneurs en AGL22 du lait au cours de 2 lactations successives n'a été publiée à notre connaissance.

Comme l'effet du numéro de lactation sur la lipolyse spontanée n'a pas été mis en évidence d'une lactation à la suivante, la répétabilité a été étudiée sans tenir compte de ce facteur. Par contre, nous avons pris en compte à la fois le stade physiologique (de lactation et de gestation) et le niveau de production des animaux, qui sont des facteurs de variation importants. En effet, la lipolyse augmente après 12 semaines et surtout après 32 semaines de lactation, sous l'effet conjugué du stade de gestation avancé (plus de 24 semaines) et du faible niveau de production (moins de $5 \mathrm{~kg}$ à la traite du soir) 
(Chazal et Chilliard, 1986, 1987a), et ces résultats ont en outre été confirmés en 1985 sur le troupeau de La Côte-Saint-André (résultats non publiés).

Ainsi, en milieu de lactation, en absence d'effet du stade de gestation (3 troupeaux), et en fin de gestation à niveau de production comparable (La Côte-Saint-André) la lipolyse semble être assez répétable chez un même animal entre 2 lactations consécutives. La teneur en AGL22 du lait n'est pas corrélée avec le nombre de cellules, ni avec les taux de lactose, de protéines ou de matières grasses du lait (résultats non publiés). Sa répétabilité n'est donc pas due à des différences d'état sanitaire de la mamelle entre vaches.

L'étude de la répétabilité de l'augmentation de la lipolyse au cours de la gestation sur une partie des vaches de La Côte-Saint-André montre la coexistence d'un effet répétable d'une lactation à l'autre, et d'un effet non reproduit la deuxième année et lié probablement aux conditions d'élevage la première année. En effet, en 1984, les teneurs en AGL22 du lait des vaches d'un premier groupe tari en juin-juillet, recevant de l'ensilage d'herbe (dactyle) en fin de gestation, augmentaient plus fortement que celles des vaches d'un deuxième groupe tari en août-septembre recevant de l'ensilage de maïs (CHAZAL et ChIlliard, 1986). Les 6 vaches du groupe 4 dont la lipolyse augmente en fin de gestation en 1984 et non en 1985 faisaient partie du premier groupe. Ceci renforcerait donc nos premières suggestions sur un effet favorisant de l'ensilage d'herbe sur la lipolyse spontanée en fin de gestation (CHAZAL et Chilliard, 1986 ; Chazal et al., 1987). Toutefois, en 1985, les animaux ont reçu soit de l'ensilage d'herbe (Ray-Grass Italien) seul ou au pâturage, soit de l'ensilage de maïs et de l'ensilage de luzerne, et cette année aucun effet « régime » sur la lipolyse n'a été observé.

Il existe chez la chèvre un effet race et un effet génétique intrarace, qui peuvent expliquer la répétabilité d'une lactation à l'autre de la lipolyse spontanée chez un même animal (BaKke et al., 1977 ; Chilliard, 1982). Par contre, chez la vache, aucun effet race n'a été mis en évidence (Herrington et Krukovsky, 1939 ; Roahen et Sommer, 1940 ; Chazal et Chilliard, 1987b). Bien qu'un effet génétique intra race ait été suggéré par DeETH et FiTzGerald (1976), ceci n'a pas été confirmé dans l'étude de Winter et al. (1981) sur 4 groupes de vaches demi-sœurs, ni sur deux paires de jumelles monozygotes. Dans le présent essai, il n'est malheureusement pas possible de rechercher un effet génétique ( 23 pères différents pour 35 vaches).

Des études sur de grands effectifs selon des protocoles appropriés seraient donc utiles à mettre en place afin de déterminer si la répétabilité de la lipolyse d'une lactation à la suivante est liée à des caractéristiques héritables des vaches, ce qui pourrait avoir des conséquences pratiques pour la sélection des reproducteurs.

\section{Conclusion}

Les résultats de cette étude doivent être considérés comme des tendances, compte tenu des limitations méthodologiques liées à la multiplicité des facteurs qui agissent sur la lipolyse spontanée (troupeau, stade physiologique, niveau de 
production, régime, année, âge...). Ils constituent toutefois un ensemble de données nouvelles par rapport à une bibliographie très pauvre dans ce domaine. Ils montrent en particulier l'existence d'une répétabilité de la lipolyse spontanée d'une lactation à la suivante, qui explique une part importante (de 30 à $40 \%$ ) de la variabilité de ce paramètre en pleine lactation, lorsque l'effet du stade de gestation est faible. Ils suggèrent également la coexistence des effets d'un facteur individuel intrinsèque répétable d'une lactation à l'autre, et d'un facteur extrinsèque dépendant des conditions d'élevage, sur l'augmentation de la lipolyse en fin de gestation. L'effet du numéro de lactation sur la lipolyse demande par ailleurs à être précisé, notamment en fin de gestation, et chez un même animal au cours des lactations successives.

Reçu le 7 juillet 1986.

Accepté pour publication le 17 mars 1987.

\section{Remerciements}

Nous remercions J.P. JULLIEN, J.P. GaRel, A. OLLIER et J. DemUle pour la conduite des troupeaux expérimentaux de La Cóte-Saint-André, de Marcenat, de Theix et de Bourg-en-Bresse, G. Bovquet, F. Trincat (GIE Rhône-Alpes), F. Gigniac et C. PORTE (ITEB) pour les dosages d'acides gras libres du lait et M.C. SINSARD (ITEB) pour la dactylographie du manuscrit. Cette étude a été réalisée grâce au concours financier du Ministère de la Recherche et de la Technologie, et de l'Insitut Technique de l'Elevage Bovin.

\section{Références bibliographiques}

AHRNÉ L., BJöRCK L., 1985. Lipolysis and the distribution of lipase activity in bovine milk in relation to stage of lactation and time of milking. J. Dairy Res., 52, 55-64.

Bakke H., Steine S., Eggum A., 1977. Flavour score and content of free fatty acids in goat milk. Acta Agric. Scand., 27, 245-249.

Chazal M.P., Cartier P., Chilliard Y., Fléchet J., Bauchart D., Duboisset F., Meyer M., 1984. Dosage des acides gras libres du lait par la méthode BDI. Cah. Techn. INRA, 6, 17-27.

Chazal M.P., Chilliard Y., 1986. Effect of stage of lactation, stage of pregnancy, milk yield and herd management on seasonal variation in spontaneous lipolysis in bovine milk. $J$. Dairy Res., $53,529-538$.

Chazal M.P., Chilliard Y., 1987a. Effets respectifs du stade de lactation, du stade de gestation et du niveau de production sur la lipolyse spontanée du lait de vache. Lait, 67, 379-392.

Chazal M.P., Chilliard Y., 1987b. Effect of breed of cow (Friesian and Montbéliarde) on spontaneous and induced lipolysis in milk. J. Dairy Res., 54, 7-11.

Chazal M.P., Chilliard Y., Coulon J.B., 1987. Effects of nature of forage on spontaneous lipolysis in milk from cows in late lactation. J. Dairy Res., 54, 13-18.

Chilliard Y., 1982. Variations physiologiques des activités lipasiques et de la lipolyse spontanée dans les laits de vache, de chèvre et de femme ₹ Revue bibliographique. Lait, 62, 1-31 et 126154.

Chilliard Y., Morand-Fehr P., 1978. Variations physiologiques de l'activité lipoprotéine-lipasique du lait de chèvre. Lait, 58, 1-16. 
Connolly J.F., 1978. Recent findings on lipolysis in Ireland. The impact of environment and nutrition on free fatty acids and flavour in milk and dairy products. 20th. Int. Dairy Congr., Doc. $62 S T(B), 32$ p.

Deeth H.C., Fitz-Gerald C.H., 1976. Lipolysis in dairy products : a review. Aust. J. Dairy Technol., 31, 53-64.

Herrington B.L., Krukovsky V.N., 1939. Studies of lipase action. III - Lipase action in the milk of individual cows. J. Dairy Sci., 22, 149-152.

Hunter A.C., Wilson J.M., Greig G.W., 1968. Spontaneous rancidity in milk from individual cows. J. Soc. Dairy Technol., 21, 139-144.

Jellema A., 1975. Note on susceptibility of bovine milk to lipolysis. Neth. Milk Dairy J., 29, 145152.

Kielsznia R., KRolikowsKa E., 1982. Influence of various factors upon the lipolytic changes in cow's milk. 2l Congr. Int. Lait, 1 (2), 145-146.

Kuzdzal-Savoie S., Mocquot G., 1960. Observations sur les qualités organoleptiques du lait. Ann. Technol. Agric., 9, 5-52.

Ortiz M.J., Kesler E.M., Watrous G.H. Jr., Cloninger W.H., 1970. Effect of the cow's body condition and stage of lactation on development of milk rancidity. J. Milk Food Technol., 33, 339-342.

Roahen D.C., Sommer H.H., 1940. Lipolytic activity in milk and cream. J. Dairy Sci., 23, 831841.

Salih A.M.A., Anderson M., 1979. Observations on the influence of high cell count on lipolysis in bovine milk. J. Dairy Res., 46, 453-462.

SEebeck R.M., 1973. The effect of body weight loss on the composition of Braham cross and Africander cross steers. I. Empty body weight, dressed carcass weight and offal components. J. Agric. Sci., 80, 201-210.

Tallamy P.T., Randolph H.E., 1969. Influence of mastitis on properties of milk. IV. Hydrolytic rancidity. J. Dairy Sci., 52, 1569-1572.

Tarassuk N.P., Yaguchi M., 1958. Effect of mastitis on susceptibility of milk to lipolysis. J. Dairy Sci., 41, 1482 (Abstract).

Winter R., Pabst K., Ordolff D., Gravert H.O., 1981. Tierspezifische Lipaseaktivität in der Kuhmilch. Kiel. Milchwirtsch. Forsch. Ber., 33, 221-226. 